\title{
Differences in Real-World Health and Economic Outcomes Among Patients with COPD Treated with Combination Tiotropium/Olodaterol Versus Triple Therapy
}

\author{
Swetha R. Palli, MS; Monica Frazer, PhD; Mary DuCharme, MLIS; Ami R. Buikema, MPH; \\ Amy J. Anderson, MS; and Jessica Franchino-Elder, PhD
}

\begin{abstract}
BACKGROUND: The 2018 Global Initiative for Chronic Obstructive Lung Disease (GOLD) recommends combination long-acting muscarinic antagonists/long-acting beta2-agonists (LAMA+LABA) as preferred maintenance therapy for patients with symptomatic chronic obstructive lung disease (COPD) after monotherapy and stepping up to triple therapy (TT; LAMA + LABA + inhaled corticosteroids [ICS]) in case of further exacerbations. Restrictions on TT recommendations have primarily been driven by higher pneumonia risk associated with regular ICS use. Evidence suggests that TT is overprescribed, which may affect economic and clinical outcomes.
\end{abstract}

OBJECTIVE: To compare health plan-paid costs, COPD exacerbations, and pneumonia diagnoses among patients newly treated with a LAMA+LABA regimen composed of tiotropium $(T I O)+$ olodaterol $(O L O)$ in a fixed-dose combination inhaler (TIO + OLO) or TT in a U.S. Medicare Advantage Part D insured population.

METHODS: This retrospective study identified COPD patients aged $\geq 40$ years who were initiating TIO + OLO or TT (index regimen) between January 1, 2014, and March 31, 2018, from a national administrative claims database. Continuous insurance coverage for 12 months pretreatment (baseline) and $\geq 30$ days posttreatment (follow-up) was required. Patients were followed until the earliest of study end (May 31, 2018), discontinuation of index regimen ( $\geq 60$-day gap in index regimen coverage), switch to a different regimen, or health plan disenrollment. Before analysis of outcomes, TIO + OLO and TT patients were 1:1 propensity score-matched on baseline demographics, comorbidities, COPD medication use, medical resource use, and costs. Cohort differences in post-match outcomes were assessed by Wald Z-test (annualized costs) and Kaplan-Meier method (time to first COPD exacerbation and pneumonia diagnosis).

RESULTS: After matching, each cohort had 1,454 patients who were well balanced on baseline characteristics. Compared with TT, the TIO + OLO cohort incurred $\$ 7,041$ (41.1\%) lower mean COPD-related total costs $(\$ 10,094$ vs. $\$ 17,135 ; P<0.001)$; cohort differences in the medical component $(\$ 3,666$ lower for TIO + OLO) were driven by lower mean acute inpatient costs $(\$ 3,053$ lower for TIO + OLO). Combined mean COPD plus pneumonia-related medical costs were $\$ 5,212(39.0 \%)$ lower for TIO + OLO versus TT $(\$ 8,209$ vs. $\$ 13,421 ; P=0.006)$, and total mean all-cause costs were $\$ 9,221$ (30.4\%) lower for TI0 + 0L0 versus TT ( $\$ 21,062$ vs. $\$ 30,283 ; P<0.001)$. Kaplan-Meier analysis found longer time to first severe COPD exacerbation $(P=0.020)$ and first pneumonia diagnosis $(P=0.002)$ for $\mathrm{TIO}+0 \mathrm{LO}$ versus TT and a lower percentage of TIO+OLO patients experiencing these events (severe COPD exacerbation: $9.0 \%$ vs. $16.1 \%$; pneumonia: $14.5 \%$ vs. $19.3 \%$ ). A secondary analysis, which expanded the $\mathrm{TIO}+0 \mathrm{LO}$ cohort to include any LAMA + LABA regimen, had similar findings for all outcomes.
CONCLUSIONS: COPD patients initiating TIO + OLO incurred lower costs to health plans and experienced fewer COPD exacerbation and pneumonia events relative to TT. These findings provide important real-world economic and clinical insight into the GOLD recommendations for TIO + OLO and LAMA + LABA therapy. The study findings also indicate the continued inconsistency between the recommendations and real-world clinical practices pertaining to TT.

J Manag Care Spec Pharm. 2020;26(10):1363-74

Copyright $\odot 2020$, Academy of Managed Care Pharmacy. All rights reserved.

\section{What is already known about this subject}

Combination long-acting muscarinic antagonists (LAMA) plus long-acting beta2-agonists (LABA) are guideline-recommended as maintenance therapy for patients with symptomatic chronic obstructive pulmonary disease (COPD) after monotherapy. Given the higher risk of pneumonia with regular inhaled corticosteroid (ICS) use, the addition of ICS to LAMA + LABA, thereby stepping up to triple therapy (TT), is recommended only for the most severe patients who continue to experience exacerbations. TT is commonly overprescribed to less severe patients in clinical practice, which may affect economic and clinical outcomes; however, previous research comparing LAMA + LABA versus TT in a real-world setting in a robust manner is limited.

\section{What this study adds}

This analysis compared outcomes for patients newly treated with tiotropium ( $\mathrm{TIO})+$ olodaterol (OLO), a fixed-dose combination of LAMA+LABA in a single inhaler, with TT in a U.S. Medicare Advantage Part D insured population.

This study adds to previous research comparing LAMA+LABA in a real-world setting by examining both health and economic outcomes in the same study and focusing on the period when patients remained on treatment.

Compared with TT, TIO+OLO-treated patients incurred $41.1 \%$ lower annualized health plan-paid costs for COPD-related services and had a lower percentage of patients with both severe COPD exacerbations and pneumonia diagnoses while taking a significantly longer time to their respective first occurrences. 


\section{Differences in Real-World Health and Economic Outcomes Among Patients with COPD Treated with Combination Tiotropium/Olodaterol Versus Triple Therapy}

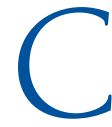
hronic obstructive pulmonary disease (COPD) has been diagnosed in $5.9 \%$ of the U.S. adult population ( 15.9 million individuals) and imposes significant clinical and economic burdens on patients, payers, and health care systems. ${ }^{1-4}$ The Global Initiative for Chronic Obstructive Lung Disease (GOLD) report categorizes patients with COPD for pharmacologic management according to exacerbation risk and symptom burden: Group A (low exacerbation risk/ low symptom burden), Group B (low exacerbation risk/high symptom burden), Group C (high exacerbation risk/low symptom burden), and Group D (high exacerbation risk/high symptom burden). ${ }^{5}$ The 2018 GOLD report (referenced during the current study execution) recommends dual therapy with long-acting muscarinic antagonists (LAMAs) plus long-acting beta2-agonists (LABAs) for patients in Groups B-D who have persistent symptoms and/or exacerbations on LAMA or LABA monotherapy. Among patients in Group C who continue to experience exacerbations, the addition of inhaled corticosteroids (ICSs) to LABA (LABA + ICS) may be also be considered. ${ }^{5}$ Finally, triple therapy (TT), consisting of LAMA+LABA+ICS, is only recommended for Group D patients and as an escalation step from LAMA + LABA (or LABA+ ICS). ${ }^{5}$

Recommendations for TT use have been restricted due to risk/benefit considerations given the substantially higher risk of pneumonia associated with ICS use in COPD patients ${ }^{6}$; a meta-analysis of clinical trials found a $53 \%$ higher risk of pneumonia for TT versus LAMA + LABA. ${ }^{7}$ The latest (2020) GOLD report further limits recommendations for ICS-containing regimens as an initial therapy to Group D patients with a history of or with concomitant asthma or for the minority with elevated eosinophil blood counts, a potential but unconfirmed biomarker for ICS responsiveness in patients with persistent exacerbations. $^{8-11}$ Despite these recommendations, several studies have reported widespread use of TT among patients in GOLD Groups A-C, thereby unnecessarily exposing patients to an elevated risk of pneumonia, wasting scarce health care resources, and contributing to the ever-escalating cost burden. ${ }^{12-16}$

Emerging real-world evidence suggests advantages of LAMA + LABA over TT. A study of patients in clinical practice reported a reduced rate of exacerbations with LAMA+LABA versus TT. ${ }^{17}$ Studies examining economic outcomes found substantial cost savings among patients initiating LAMA + LABA, specifically as tiotropium (TIO) + olodaterol $(\mathrm{OLO})^{18}$ in a fixeddose combination inhaler, relative to $\mathrm{TT}^{19-21}$ Our previous study examined clinical and economic outcomes for TIO + OLO versus TT and found that initiating $\mathrm{TIO}+\mathrm{OLO}$ relative to TT was associated with 35\% lower annual COPD-related health plan-paid costs and a smaller proportion of patients experiencing severe COPD exacerbations and pneumonia occurrences. ${ }^{21}$ While these results provided important insight into the differential burden of TIO + OLO versus TT, they were limited by the intent-to-treat approach, which observed patients for the entire first year after therapy initiation, irrespective of subsequent therapy changes that are relatively common in clinical practice. ${ }^{22,23}$ Further, requiring 12 months of followup may lead to selection of healthier cohorts, increasing the survival bias if a treatment is associated with survival. ${ }^{24}$ This study sought to address these limitations while expanding our previous findings by examining outcomes for the duration that patients remained on their initial regimen. We also leveraged the availability of additional data and refined the assessment of the burden attributable to pneumonia.

The primary objective of this study was to compare health care costs, exacerbations, and pneumonia outcomes among patients newly treated with TIO+OLO versus TT in a real-world setting. The secondary objective extended the $\mathrm{TIO}+\mathrm{OLO}$ cohort to patients treated with any LAMA+LABA versus TT. Consistent with our previous study, we focused on patients with managed Medicare insurance because Medicare incurs the largest share of COPD-related costs in the United States, ${ }^{4,25}$

\section{Methods}

\section{Design and Data Source}

This retrospective study used administrative claims data from January 1, 2013-May 31, 2018 from the Optum Research Database (ORD). The ORD contains enrollment information and medical and pharmacy claims submitted to each patient's health plans for reimbursement. Medical claims include International Classification of Diseases, Ninth Revision, Clinical Modification (ICD-9-CM) and International Classification of Diseases, Tenth Revision, Clinical Modification (ICD-10-CM) diagnosis and procedure codes and site of service codes. Pharmacy claims include National Drug Code numbers for medications dispensed with quantity, dose, and number of days supply. The ORD is geographically diverse in coverage and represented approximately $21 \%$ ( 8.3 million individuals) of the U.S. population, with Medicare Advantage prescription drug (MAPD) plan benefits in 2018. No identifiable protected health information was extracted or accessed during the course of the study, and all data were accessed in compliance with the Health Insurance Portability and Accountability Act's rules; therefore, institutional review board approval or waiver of authorization was not required.

\section{Patient Selection and Cohort Assignment}

The first step in the selection process was to identify patients initiating a COPD maintenance therapy (LAMA monotherapy, LABA+ICS, LAMA+LABA, or TT) between January 1, 2014, and March 31, 2018 (identification period) using pharmacy claims. LABA monotherapy was not considered because of its infrequent usage ( $<3 \%$ of monotherapy patients). ${ }^{26}$ Index regimen was the first of the above 4 regimens observed for 


\section{Differences in Real-World Health and Economic Outcomes Among Patients with COPD Treated with Combination Tiotropium/Olodaterol Versus Triple Therapy}

$\geq 30$ consecutive days, without other overlapping nonindex maintenance regimens (therefore mutually exclusive). Patients initiating LAMA + LABA or TT were retained for this analysis. The index date for patients initiating single inhaler regimens, such as TIO+OLO, was the first pharmacy claim during the identification period. For multiple inhaler regimens, including open LAMA+LABA and most patients initiating on TT, the index date was defined as the start of $\geq 30$ consecutive days of overlapping supply of LAMA and LABA—and for TT, ICS— components (free or fixed-dose combinations).

The diagnosis of COPD was established by ICD-9-CM (491.xx-493.2x and 496.xx) or ICD-10-CM (J41-J44) codes on $\geq 2$ medical claims on separate dates of service during the study period (January 1, 2013-May 31, 2018). Additional inclusion criteria were patients aged $\geq 40$ years as of the index date, complete demographic information availability, and $\geq 13$ months of continuous medical and pharmacy enrollment in an MAPD health plan involving 12 months of pre-index (baseline period) and $\geq 1$ month of post-index (follow-up period) data. Patients with $\geq 2$ medical claims on separate dates of service involving a diagnosis of asthma, lung cancer, or cystic fibrosis during the study period and evidence of the index regimen during the baseline period were excluded. ${ }^{21}$

The primary analysis included COPD patients who were initiating treatment with only $\mathrm{TIO}+\mathrm{OLO}$ (and no other LAMA+LABA) or TT. The secondary analysis, comparing LAMA+LABA versus TT, used the same patient selection criteria as the primary analysis but expanded the TIO + OLO cohort to include patients whose index regimen was any LAMA+LABA (free or fixed-dose combinations, including $\mathrm{TIO}+\mathrm{OLO})$.

\section{Follow-up Period}

Patients were followed until the earliest occurrence of index regimen discontinuation, switch to a nonindex regimen, health plan disenrollment, or end of the study period (May 31, 2018). Discontinuation was defined as a gap of $\geq 60$ days between pharmacy fills of the index regimen. The discontinuation date was the last prescription runout date (fill-date + days supply of the index regimen for single inhalers or overlapping therapy in the case of multiple inhalers) prior to the occurrence of $\geq 60$-day gap. Switch was defined as a change to a nonindex regimen for $\geq 30$ consecutive days.

\section{Measures}

Patient Characteristics. Demographic characteristics were determined on the index date. Clinical characteristics including Quan-Charlson comorbidity and Elixhauser scores were calculated during the 12 -month baseline period. ${ }^{27,28}$ COPD maintenance and rescue medication use was ascertained from pharmacy claims. Severe COPD exacerbations were defined as an inpatient admission with a COPD diagnosis code in the primary position. Moderate exacerbations required either an emergency department (ED) visit with a COPD primary diagnosis or an ambulatory visit with a COPD diagnosis code in any position plus a pharmacy claim for an oral corticosteroid (OCS) or COPD guideline-recommended antibiotic prescription within 7 days following the ambulatory visit. Exacerbations occurring within 14 days of each other were considered a single exacerbation episode and were classified as per the highest severity. Patients meeting GOLD C/D criteria (i.e., those at high risk of exacerbations $[\geq 2$ moderate and/or $\geq 1$ severe]) during the baseline period were flagged for further examination. ${ }^{5}$

Outcomes. Follow-up costs were determined from medical and pharmacy claims beginning on the index date. Medical costs included costs for medical service use and were categorized as acute inpatient, ED, ambulatory (office and outpatient), and other (e.g., durable medical equipment, home health visits, independent laboratory tests, long-term care). All-cause total costs were the sum of all medical costs for any medical service plus all outpatient pharmacy costs. COPD-related total costs included the sum of costs of all medical claims with a diagnosis of COPD in any position plus outpatient pharmacy claims for COPD medications (maintenance, rescue, OCSs, and antibiotics). COPD-attributable total costs, a subset of COPD-related total costs, were the sum of medical claims with a COPD diagnosis in the primary position only plus outpatient pharmacy claims for COPD-related treatment. Pneumoniarelated medical costs included medical claims with a diagnosis code for pneumonia and/or acute bronchitis/bronchiolitis in any position (codes previously reported). ${ }^{21} \mathrm{COPD} /$ pneumoniarelated medical cost burden included the costs of claims involving a diagnosis for COPD, pneumonia, and/or acute bronchitis/bronchiolitis.

To examine the incremental burden of pneumonia to COPD-related cost, non-COPD pneumonia-related costs were estimated by accounting for pneumonia-related claims and excluding those with a COPD diagnosis on the same claim. To avoid skewing of means by patients with extremely short or long follow-ups, all cost outcomes captured during the variable follow-up period were annualized at the cohort level by summing the total costs for all patients and dividing by the sum total days on treatment for all patients and then multiplying the result by 365 . All costs measures were computed from health plan-paid costs and inflation-adjusted to 2017 U.S. dollars. ${ }^{29}$ Additional outcomes, including assessment of pneumonia diagnoses and exacerbations (as defined above) during follow-up, started on the day after the index date.

\section{Propensity Score Matching and Statistical Analysis}

Patients in the TIO+OLO and TT cohorts were matched 1:1 on baseline characteristics using propensity score nearest 


\section{Differences in Real-World Health and Economic Outcomes Among Patients with COPD Treated with Combination Tiotropium/Olodaterol Versus Triple Therapy}

neighbor matching (PSM), within a caliper of 0.001 of the standard deviation of the estimated logit of the propensity score. Iterative modeling was conducted to identify the logistic model that met the caliper of 0.001. Pre-PSM cohort differences in baseline characteristics were analyzed by Pearson Chisquare test and 2-sample t-test for categorical and continuous measures, respectively. Post-PSM baseline characteristics and cost outcomes were analyzed by Rao-Scott test (categorical measures) and Wald Z-test using robust standard errors in an ordinary least squares regression (continuous measures). Kaplan-Meier analyses were conducted to examine time to first COPD exacerbation and first pneumonia diagnosis; cohort differences were analyzed with the log-rank test. For key outcomes, the number needed to treat (NNT) of TT patients who would instead need to be treated with TIO/OLO to avoid 1 event was calculated. Rates were derived from the annualized Kaplan-Meier analyses.

The secondary analysis (LAMA+ LABA vs. TT) repeated the same PSM process and statistical analysis methods used in the primary analysis.

\section{Results}

\section{Study Sample and Patient Characteristics}

A total of 1,592 TIO + OLO and 7,310 TT patients initiated their index regimen from January 1, 2014-March 31, 2018 (Supplemental Figure 1, available in online article). After matching, 1,454 patients remained in each cohort.

Before matching, the TIO + OLO and TT cohorts were similar in mean age ( 72.3 vs. 72.5 years) but differed significantly for most other characteristics (Table 1). The TT cohort had a higher comorbidity burden, a greater percentage of patients with exacerbations and pneumonia and use of most COPD medications, and higher mean costs. Post-PSM, cohorts were well balanced for all of these characteristics as evidenced by a standardized difference $<10 \%$ and significance values of $P>0.05$. The only remaining significant differences between cohorts after matching were a slightly higher percentage of patients in the TT cohort with a high exacerbation risk (standardized difference $=6.9 \%$ ), any exacerbation history (standardized difference $=9.0 \%$ ), and counts of any exacerbation in the TT cohort, which were driven by the rate of moderate exacerbations (standardized difference $=10.5 \%$ ). The mean time on index treatment was longer for $\mathrm{TIO}+\mathrm{OLO}$ versus TT (134 vs. 108 days; $P<0.001$ ). The most common index TT regimen was a LABA/ICS combination (95.9\%) with LAMA monotherapy (95.5\%).

\section{Outcomes}

Costs. Patients treated with TIO+OLO incurred $\$ 7,041$ (41.1\%) lower mean annualized COPD-related total costs ( $\$ 10,094$ vs. $\$ 17,135 ; P<0.001$; Figure 1$)$. The medical component of COPD-related costs was lower in the TIO + OLO cohort by an average of $\$ 3,666(\$ 6,449$ vs. $\$ 10,115 ; P=0.002)$ and was driven primarily by lower mean acute inpatient costs $(\$ 3,053)$. Lower mean COPD-related pharmacy costs in the TIO + OLO cohort accounted for the balance of the costs savings (mean cohort difference $=\$ 3,374$ ).

After accounting for pneumonia, COPD/pneumonia-related medical costs were lower by $\$ 5,212$ for $\mathrm{TIO}+\mathrm{OLO}$ versus $\mathrm{TT}$, averaging $\$ 8,209$ and $\$ 13,421$ for the TIO+OLO and TT cohorts, respectively (Figure 2). Thus, the incremental medical burden when allowing for pneumonia was $\$ 1,760$ (or $27.3 \%$ ) for TIO + OLO and $\$ 3,306$ for TT (or 32.7\%) higher than their respective mean COPD-related medical costs. COPD-attributable costs were also lower for $\mathrm{TIO}+\mathrm{OLO}$ vs. TT, with between-cohort differences averaging $\$ 1,879$ for medical costs ( $\$ 2,968$ vs. $\$ 4,847 ; P=0.038)$ and $\$ 3,374$ for pharmacy costs $(\$ 3,646$ vs. $\$ 7,020 ; P<0.001)$, leading to a difference of $\$ 5,253$ in total costs $(\$ 6,614$ vs. $\$ 11,867 ; P<0.001$; data not shown). Mean annualized all-cause total costs were lower by $\$ 9,221$ (30.4\%) for TIO + OLO versus TT (\$21,062 vs. \$30,283; $P<0.001)$

COPD Exacerbations. The Kaplan-Meier analysis revealed that time to first severe COPD exacerbation was longer for $\mathrm{TIO}+\mathrm{OLO}$ versus TT $(P=0.020$; Figure 3$)$; the percentage of patients with severe exacerbation at 1 year was $9.0 \%$ for $\mathrm{TIO}+\mathrm{OLO}$ versus $16.1 \%$ for TT (i.e., NNT = 14). There was no significant difference between cohorts in the Kaplan-Meier distribution for any (i.e., moderate or severe) COPD exacerbation ( $44.5 \%$ vs. $51.2 \%$; $P=0.162$ ).

Pneumonia. Time to first pneumonia diagnosis was longer for $\mathrm{TIO}+\mathrm{OLO}$ vs. TT $(P=0.002)$. At 1 year, $14.5 \%$ of TIO $+\mathrm{OLO}$ vs. $19.3 \%$ of TT patients were diagnosed with pneumonia $(\mathrm{NNT}=21)$. There was also a longer time to first diagnosis of pneumonia or acute bronchitis/bronchiolitis ( $P=0.002$; Figure 4), with a lower percentage of $\mathrm{TIO}+\mathrm{OLO}$ versus TT patients diagnosed per annum (22.8\% vs. $28.4 \%$; NNT $=18)$.

Secondary Analysis. The secondary analysis included 3,845 matched patients each in the LAMA+LABA and TT cohorts (Supplemental Figure 1, available in online article). After PSM, standardized differences were $<10 \%$ for all baseline characteristics, and the only significant difference between cohorts was a slightly higher percentage of LAMA+LABA patients treated with rescue medications or OCSs at baseline (Supplemental Table 1, available in online article).

After matching, the LAMA+LABA cohort primarily represented patients initiating umeclidinium + vilanterol (69.4\%) followed by $\mathrm{TIO}+\mathrm{OLO}$ (23.3\%); the most common index TT regimen was an ICS/LABA combination (95.9\%)+ LAMA monotherapy (95.6\%). Duration of treatment averaged 140 versus 103 days for the LAMA + LABA and TT cohorts, respectively $(P<0.001)$. 


\section{Differences in Real-World Health and Economic Outcomes Among Patients with COPD Treated with Combination Tiotropium/Olodaterol Versus Triple Therapy}

\section{TABLE 1 Baseline Demographic and Clinical Characteristics and Follow-up Treatment for TIO + OLO and}

TT Cohorts Before and After Propensity Score Matchinga

\begin{tabular}{|c|c|c|c|c|c|c|c|c|c|c|c|}
\hline \multirow{3}{*}{$\begin{array}{l}\text { Baseline Characteristic } \\
\text { Age, years, mean (SD) }\end{array}$} & \multicolumn{5}{|c|}{ Before 1:1 PSM } & \multicolumn{6}{|c|}{ After 1:1 PSM } \\
\hline & \multicolumn{2}{|c|}{$\begin{array}{l}\mathrm{TIO}+\text { OLO } \\
(\mathrm{n}=1,592)\end{array}$} & $\begin{array}{c}\text { TT } \\
(\mathrm{n}=7,310)\end{array}$ & \multirow{2}{*}{$\begin{array}{c}\text { Std Diff } \\
\% \% \\
-2.12\end{array}$} & \multirow{2}{*}{$\frac{P \text { Value }}{0.440}$} & \multicolumn{2}{|c|}{$\begin{array}{l}\mathrm{TIO}+\mathrm{OLO} \\
(\mathrm{n}=1,454)\end{array}$} & \multicolumn{2}{|c|}{$\begin{array}{c}\text { TT } \\
(\mathrm{n}=1,454)\end{array}$} & \multirow{2}{*}{$\begin{array}{c}\text { Std Diff } \\
\% \\
-0.20\end{array}$} & \multirow{2}{*}{$\frac{P \text { Value }}{0.956}$} \\
\hline & 72.3 & $(8.6)$ & $72.5 \quad(8.5)$ & & & 72.2 & $(8.7)$ & 72.2 & $(8.4)$ & & \\
\hline \multicolumn{12}{|l|}{ Age group, years, \% (n) } \\
\hline $40-64$ & 16.8 & $(268)$ & $16.0(1,172)$ & 2.16 & 0.431 & 17.3 & $(251)$ & 17.1 & $(249)$ & 0.36 & 0.919 \\
\hline $65-74$ & 42.4 & $(675)$ & $43.0(3,145)$ & -1.26 & 0.649 & 42.9 & $(623)$ & 42.1 & $(612)$ & 1.53 & 0.668 \\
\hline$\geq 75$ & 40.8 & $(649)$ & $40.9(2,993)$ & -0.36 & 0.896 & 39.9 & $(580)$ & 40.8 & $(593)$ & -1.82 & 0.615 \\
\hline Male, \% (n) & 52.6 & $(837)$ & $48.8(3,568)$ & 7.54 & 0.006 & 52.5 & $(763)$ & 53.1 & $(772)$ & -1.24 & 0.736 \\
\hline \multicolumn{12}{|l|}{ U.S. census region, \% (n) } \\
\hline Northeast & 12.4 & $(198)$ & $20.6(1,509)$ & -22.22 & $<0.001$ & 13.3 & (193) & 13.8 & $(200)$ & -1.41 & 0.700 \\
\hline Midwest & 22.2 & $(354)$ & $29.2(2,132)$ & -15.91 & $<0.001$ & 23.6 & (343) & 25.0 & $(364)$ & -3.37 & 0.344 \\
\hline South & 59.6 & $(948)$ & $41.9(3,060)$ & 35.94 & $<0.001$ & 56.9 & $(827)$ & 54.8 & $(797)$ & 4.16 & 0.221 \\
\hline West & 5.8 & $(92)$ & $8.3 \quad(609)$ & -9.98 & $<0.001$ & 6.3 & $(91)$ & 6.4 & (93) & -0.57 & 0.874 \\
\hline Charlson comorbidity score, mean (SD) & 2.3 & $(1.8)$ & $(1.9)$ & -9.51 & $<0.001$ & 2.3 & $(1.8)$ & 2.3 & $(1.8)$ & 2.31 & 0.523 \\
\hline \multicolumn{12}{|l|}{ Charlson comorbidity score categories, \% (n) } \\
\hline $0-1$ & 46.4 & $(738)$ & $44.5(3,250)$ & 3.81 & 0.168 & 47.0 & $(684)$ & 49.0 & $(713)$ & -3.99 & 0.280 \\
\hline $2-3$ & 31.9 & $(507)$ & $31.1(2,270)$ & 1.71 & 0.536 & 31.9 & $(464)$ & 29.5 & $(429)$ & 5.22 & 0.158 \\
\hline $4-5$ & 16.0 & $(254)$ & $17.5(1,278)$ & -4.10 & 0.143 & 15.5 & $(225)$ & 15.5 & $(226)$ & -0.19 & 0.959 \\
\hline$\geq 6$ & 5.8 & (93) & $7.0 \quad(512)$ & -4.74 & 0.095 & 5.6 & $(81)$ & 5.9 & $(86)$ & -1.48 & 0.682 \\
\hline Elixhauser comorbidity score, mean (SD) & 9.5 & $(9.2)$ & $10.8 \quad(9.7)$ & -13.74 & $<0.001$ & 9.5 & $(9.2)$ & 9.1 & $(8.9)$ & 4.18 & 0.249 \\
\hline Pneumonia diagnosis, \% (n) & 17.1 & $(272)$ & $27.6(2,020)$ & -25.52 & $<0.001$ & 17.9 & $(260)$ & 17.3 & $(252)$ & 1.44 & 0.682 \\
\hline $\begin{array}{l}\text { Pneumonia and/or acute bronchitis/ } \\
\text { bronchiolitis diagnosis, \% (n) }\end{array}$ & 30.0 & $(478)$ & $39.1(2,858)$ & -19.16 & $<0.001$ & 31.1 & $(452)$ & 29.0 & $(422)$ & 4.50 & 0.204 \\
\hline \multicolumn{12}{|l|}{ COPD medications, \% (n) } \\
\hline Naive to LAMA or LABAc & 92.2( & $1,468)$ & $60.0(4,387)$ & 81.55 & $<0.001$ & 91.5 & $(1,330)$ & 90.8 & $(1,320)$ & 2.42 & 0.432 \\
\hline Any LAMAd & 4.2 & $(67)$ & $20.3(1,486)$ & 50.69 & $<0.001$ & 4.6 & $(67)$ & 5.7 & (83) & -4.98 & 0.158 \\
\hline Any LABAd & 2.9 & $(46)$ & $22.3(1,629)$ & -61.14 & $<0.001$ & 3.2 & $(46)$ & 3.6 & $(52)$ & -2.29 & 0.446 \\
\hline Any ICSd & 3.8 & $(60)$ & $27.0(1,976)$ & -66.08 & $<0.001$ & 4.1 & $(60)$ & 4.5 & $(65)$ & -1.70 & 0.559 \\
\hline $\mathrm{LABA}+\mathrm{ICS}$ e & 1.8 & $(28)$ & $20.9(1,525)$ & -63.26 & $<0.001$ & 1.9 & $(28)$ & 2.1 & $(31)$ & -1.46 & 0.532 \\
\hline Any SABA ${ }^{\mathrm{d}}$ & 51.4 & $(818)$ & $59.6(4,360)$ & -16.68 & $<0.001$ & 52.3 & $(760)$ & 50.0 & $(727)$ & 4.54 & 0.216 \\
\hline Any SAMAd & 15.3 & $(244)$ & $21.4(1,565)$ & -15.76 & $<0.001$ & 16.3 & $(237)$ & 13.9 & $(202)$ & 6.73 & 0.067 \\
\hline SABA+SAMA ${ }^{\mathrm{e}}$ & 13.3 & $(212)$ & $19.1(1,397)$ & -15.77 & $<0.001$ & 14.2 & $(206)$ & 12.5 & $(181)$ & 5.06 & 0.168 \\
\hline Methylxanthines & 0.4 & (7) & $1.5 \quad(110)$ & -10.87 & $<0.001$ & 0.5 & (7) & 0.9 & (13) & -4.99 & 0.180 \\
\hline Phosphodiesterase-4 inhibitors & 0.5 & $(8)$ & $(61)$ & -4.07 & 0.171 & 0.6 & $(8)$ & 0.6 & (9) & -0.90 & 0.808 \\
\hline Oral corticosteroids & 38.7 & $(616)$ & $46.0(3,363)$ & -14.84 & $<0.001$ & 39.6 & $(575)$ & 40.6 & $(590)$ & -2.11 & 0.562 \\
\hline Oxygen therapy, \% (n) & 19.8 & $(315)$ & $33.8(2,473)$ & -32.11 & $<0.001$ & 20.9 & (304) & 22.2 & $(323)$ & -3.18 & 0.375 \\
\hline \multicolumn{12}{|l|}{ Annual exacerbations } \\
\hline \multicolumn{12}{|l|}{ Severe } \\
\hline$\%(n)$ & 8.5 & $(136)$ & $26.3(1,924)$ & -48.20 & $<0.001$ & 9.3 & $(135)$ & 8.2 & $(119)$ & 3.90 & 0.223 \\
\hline Count, mean (SD) & 0.10 & $(0.35)$ & $0.32 \quad(0.62)$ & -43.74 & $<0.001$ & 0.11 & $(0.37)$ & 0.09 & $(0.32)$ & 5.43 & 0.093 \\
\hline \multicolumn{12}{|l|}{ Moderate } \\
\hline$\%(n)$ & 36.1 & $(575)$ & $43.5(3,177)$ & -15.04 & $<0.001$ & 36.7 & $(534)$ & 41.1 & $(598)$ & -9.04 & 0.014 \\
\hline Count, mean (SD) & 0.54 & $(0.92)$ & $0.73 \quad(1.10)$ & -18.18 & $<0.001$ & 0.55 & $(0.92)$ & 0.65 & $(1.03)$ & -10.46 & 0.005 \\
\hline Any (moderate or severe) & & & & & & & & & & & \\
\hline$\%(n)$ & 40.6 & $(647)$ & $59.4(4,340)$ & -38.13 & $<0.001$ & 41.7 & $(606)$ & 46.2 & $(671)$ & -9.02 & 0.011 \\
\hline Count, mean (SD) & 0.64 & $(1.03)$ & $1.05 \quad(1.29)$ & -34.69 & $<0.001$ & 0.66 & $(1.04)$ & 0.74 & $(1.09)$ & -7.89 & 0.029 \\
\hline $\begin{array}{l}\text { GOLD C/D (i.e., high exacerbation risk), } \\
\%(n)^{f}\end{array}$ & 17.9 & $(285)$ & $38.7(2,830)$ & -47.48 & $<0.001$ & 18.8 & $(273)$ & 21.5 & (313) & -6.86 & 0.047 \\
\hline Annual acute inpatient utilization, \% (n) & & & & & & & & & & & \\
\hline COPD-related & 18.5 & $(295)$ & $41.8(3,057)$ & -52.45 & $<0.001$ & 20.1 & $(292)$ & 17.9 & $(260)$ & 5.61 & 0.058 \\
\hline COPD-attributable & 8.5 & $(135)$ & $26.3(1,920)$ & -48.29 & $<0.001$ & 9.2 & $(134)$ & 8.1 & (118) & 3.91 & 0.220 \\
\hline Pneumonia-relatedg & 9.7 & $(155)$ & $22.3(1,631)$ & -34.80 & $<0.001$ & 10.5 & $(153)$ & 11.1 & $(161)$ & -1.77 & 0.607 \\
\hline All-cause & 26.2 & $(417)$ & $46.8(3,419)$ & -43.76 & $<0.001$ & 27.9 & $(405)$ & 27.1 & (394) & 1.69 & 0.601 \\
\hline
\end{tabular}




\section{Differences in Real-World Health and Economic Outcomes Among Patients with COPD Treated with Combination Tiotropium/Olodaterol Versus Triple Therapy}

TABLE 1 Baseline Demographic and Clinical Characteristics and Follow-up Treatment for TIO+OLO and TT Cohorts Before and After Propensity Score Matchinga (continued)

\begin{tabular}{|c|c|c|c|c|c|c|c|c|}
\hline \multirow[b]{2}{*}{ Baseline Characteristic } & \multicolumn{4}{|c|}{ Before 1:1 PSM } & \multicolumn{4}{|c|}{ After 1:1 PSM } \\
\hline & $\begin{array}{l}\text { TIO + OLO } \\
(\mathbf{n}=1,592)\end{array}$ & $\begin{array}{c}\text { TT } \\
(\mathbf{n}=7,310)\end{array}$ & $\begin{array}{c}\text { Std Diff } \\
\%\end{array}$ & $P$ Value & $\begin{array}{l}\text { TIO + OLO } \\
(\mathrm{n}=1,454)\end{array}$ & $\begin{array}{c}\text { TT } \\
(\mathrm{n}=1,454)\end{array}$ & $\begin{array}{c}\text { Std Diff } \\
\%\end{array}$ & $P$ Value \\
\hline \multicolumn{9}{|c|}{ Annual health plan-paid costs, \$, mean (SD) } \\
\hline COPD-related total cost & $4,693(12,264)$ & $11,247(21,505)$ & -37.44 & $<0.001$ & $5,053(12,733)$ & $4,727(13,932)$ & 2.45 & 0.480 \\
\hline COPD-attributable total cost & $1,964 \quad(7,613)$ & $6,065(13,194)$ & -38.07 & $<0.001$ & $2,124 \quad(7,943)$ & $2,160(10,546)$ & -0.38 & 0.916 \\
\hline Pneumonia-related medical cost $\mathrm{g}$ & $1,976(10,577)$ & $4,348(15,014)$ & -18.27 & $<0.001$ & $2,125 \quad(11,014)$ & $2,231 \quad(12,073)$ & -0.92 & 0.800 \\
\hline All-cause total cost & $16,191(26,545)$ & $21,927(31,102)$ & -19.84 & $<0.001$ & $16,491 \quad(27,173)$ & $16,360(24,371)$ & -0.51 & 0.889 \\
\hline \multicolumn{9}{|l|}{ Follow-up treatment } \\
\hline \multicolumn{9}{|l|}{ Index treatment, \% (n) } \\
\hline Tiotropium/olodaterol & $100(1,592)$ & $(76)$ & $1,379.74$ & $<0.001$ & $100(1,454)$ & (17) & $1,300.23$ & - \\
\hline Umeclidinium/vilanterol & 0 & $2.9 \quad(214)$ & -24.56 & $<0.001$ & 0 & (53) & -27.51 & - \\
\hline Indacaterol/glycopyrrolate & 0 & 0 & - & - & 0 & 0 & - & - \\
\hline Glycopyrrolate/formoterol fumarate & 0 & (12) & -5.73 & 0.106 & 0 & 0.1 & -5.25 & - \\
\hline Tiotropium bromide & 0 & $89.1(6,512)$ & -403.99 & $<0.001$ & 0 & $86.9(1,263)$ & -363.66 & - \\
\hline Other LAMA & 0 & $7.2 \quad(528)$ & -39.46 & $<0.001$ & 0 & $8.7 \quad(127)$ & -43.75 & - \\
\hline $\begin{array}{l}\text { Fluticasone furoate/umeclidinium/ } \\
\text { vilanterol }\end{array}$ & 0 & (10) & -5.23 & 0.14 & 0 & 0.2 & -6.43 & - \\
\hline \multicolumn{9}{|l|}{ Index treatment class, $\%(\mathrm{n})^{\mathrm{h}}$} \\
\hline ICS monotherapy & 0 & $6.0 \quad(437)$ & -35.66 & $<0.001$ & 0 & (72) & -32.28 & - \\
\hline LABA monotherapy & (3) & $1.5 \quad(106)$ & -14.03 & $<0.001$ & 0.2 & (13) & -9.31 & 0.013 \\
\hline LAMA monotherapy & 0 & $96.2(7,032)$ & -711.27 & $<0.001$ & 0 & $95.5(1,388)$ & -648.54 & - \\
\hline LABA/ICS & 0 & $96.3(7,041)$ & -723.53 & $<0.001$ & 0 & $95.9(1,394)$ & -681.66 & - \\
\hline LAMA/LABA & $100(1,592)$ & $4.1 \quad(301)$ & 682.43 & $<0.001$ & $100(1,454)$ & $(72)$ & 619.59 & - \\
\hline $\begin{array}{l}\text { Number of inhalers on index date, } \\
\text { mean (SD) }\end{array}$ & $1.00 \quad(0.04)$ & $2.04 \quad(0.21)$ & -686.19 & $<0.001$ & $1.00 \quad(0.05)$ & $2.02 \quad(0.17)$ & -828.14 & $<0.001$ \\
\hline Treatment duration, mean days (SD) & $134.4(120.9)$ & $106.7(105.3)$ & 24.40 & $<0.001$ & $134.0(121.0)$ & $107.6(104.4)$ & 23.33 & $<0.001$ \\
\hline \multicolumn{9}{|l|}{ End of index treatment, \% (n) } \\
\hline Study end & $22.4 \quad(356)$ & $16.8(1,225)$ & 14.16 & $<0.001$ & $22.5 \quad(327)$ & $14.6 \quad(212)$ & 20.46 & $<0.001$ \\
\hline Discontinuation & $69.6(1,108)$ & $38.5(2,813)$ & 65.72 & $<0.001$ & $69.1(1,005)$ & $47.0 \quad(683)$ & 46.05 & $<0.001$ \\
\hline Treatment switch & $(83)$ & $24.7(1,805)$ & -56.78 & $<0.001$ & $(79)$ & $21.0 \quad(305)$ & -47.17 & $<0.001$ \\
\hline $\begin{array}{l}\text { Discontinue and switch to new } \\
\text { treatment, mean (SD) }\end{array}$ & $(45)$ & $20.1(1,467)$ & -52.25 & $<0.001$ & (43) & $17.5 \quad(254)$ & -49.36 & $<0.001$ \\
\hline
\end{tabular}

aPropensity scores computed from a logistic regression model adjusted for age group (<65, 65-74, $\geq 75$ years); sex; U.S. census region (Northeast, Midwest, South, West); index regimen prescriber specialty (pulmonology, all others); and baseline characteristics including pneumonia and/or acute bronchitis/bronchiolitis diagnosis; congestive heart failure diagnosis; severe COPD exacerbation; oxygen therapy; COPD medication use (any LAMA [free dose or combination inhaler], any LABA/ICS [single combination inhaler], any ICS [free dose or combination inhaler], count of COPD medication pharmacy fills [0-1, 2-3, 3-9, 210]); medical resource use (all-cause ED visit, ED visits with COPD diagnosis in the primary position, COPD-related acute inpatient stay, pneumonia-related [narrow definition] acute inpatient stay, COPD-related other resource); counts of medical resource use (all-cause ambulatory visit, all-cause office visit, all-cause acute inpatient stay, COPD-related office visit); and COPD-related medical cost quartile group $(<\$ 800, \$ 800$ to $<\$ 3,600, \$ 3,600$ to $<\$ 11,000$, and $\geq \$ 11,000)$.

${ }^{b}$ As of index date.

cBased on medical and pharmacy claims.

dFree dose or combination inhaler.

esingle combination inhaler.

$f \geq 2$ moderate exacerbations or $\geq 1$ severe exacerbation

gMedical claims with a diagnosis of pneumonia or acute bronchitis/bronchiolitis in any position that may include claims with COPD diagnosis.

hMedication classes are not mutually exclusive.

$C O P D=$ chronic obstructive pulmonary disease; $E D=$ emergency department; $G O L D=$ Global Initiative for Chronic Obstructive Lung Disease; ICS =inhaled corticosteroid; LABA = long-acting beta2 agonist; LAMA = long-acting muscarinic antagonist; PSM = propensity score matching; SABA=short-acting beta2 agonist; SAMA=short-acting muscarinic antagonist; $S D=$ standard deviation; Std Diff=standardized difference; TIO + OLO=tiotropium/olodaterol; TT = triple therapy.

Consistent with the primary analysis, the LAMA+LABA cohort had lower mean annualized costs versus TT, with differences between cohorts averaging $\$ 6,412$ for COPD-related total costs (medical and pharmacy contributions of $48.4 \%$ and $51.6 \%$, respectively), $\$ 4,258$ for COPD/pneumonia-related medical costs, $\$ 5,250$ for COPD-attributable total costs (medical and pharmacy contributions of $37 \%$ and $63 \%$, respectively), and $\$ 8,071$ for all-cause total costs (all $P<0.001$ ). 


\section{FIGURE 1 Post-Match Annualized COPD-Related Costs During Follow-up with TIO+OLO vs. TT}

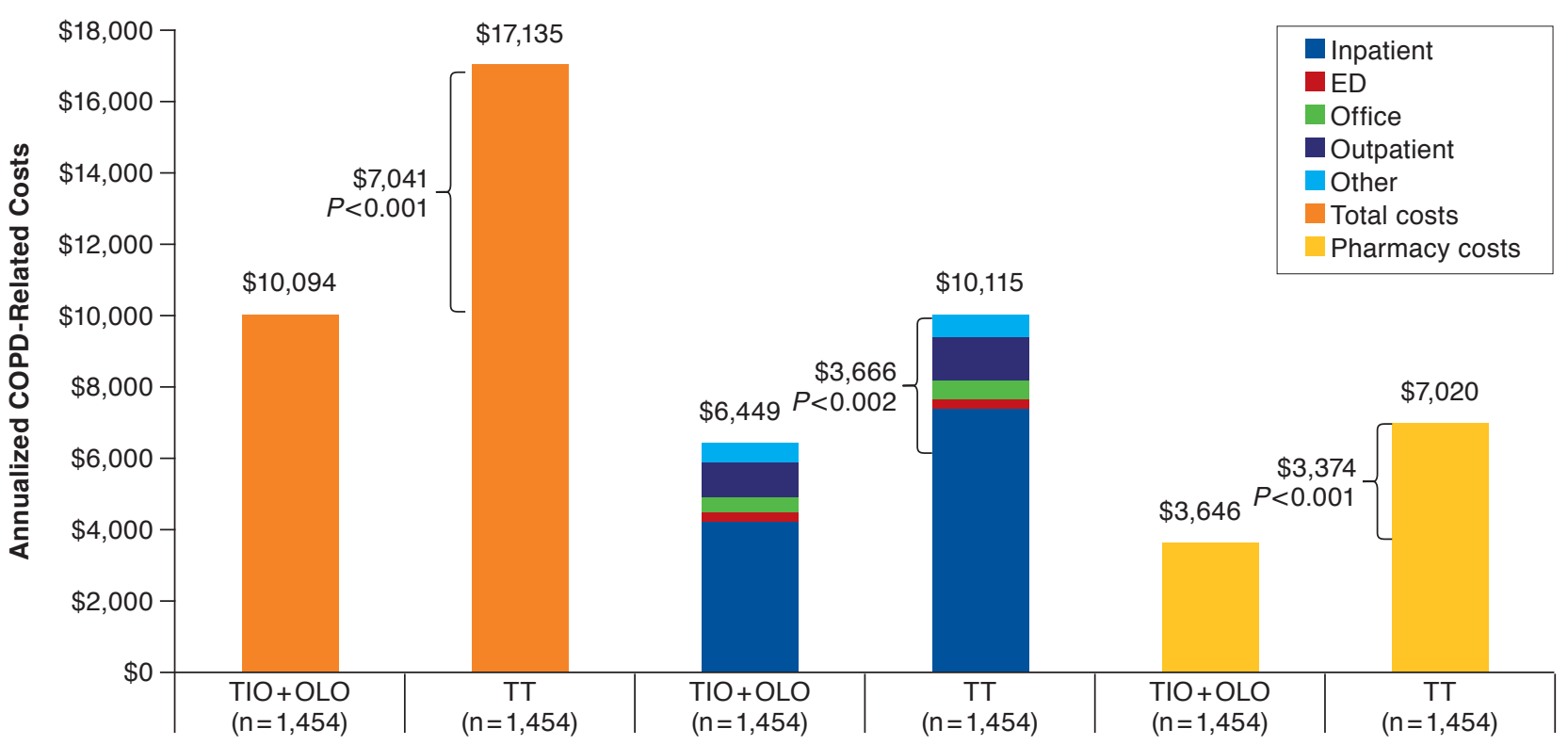

Total Costs

Medical Costs

Pharmacy Costs

\begin{tabular}{|l|c|c|c|c|c|}
\hline & \multicolumn{5}{|c|}{ Annualized Medical Cost Components } \\
\cline { 2 - 6 } & Acute Inpatient & ED & Office & Outpatient & Other \\
\hline TIO+OLO & $\$ 4,316$ & $\$ 272$ & $\$ 410$ & $\$ 941$ & $\$ 510$ \\
\hline TT & $\$ 7,370$ & $\$ 376$ & $\$ 525$ & $\$ 1,154$ & $\$ 690$ \\
\hline Difference in means & $-\$ 3,053$ & $-\$ 104$ & $-\$ 116$ & $-\$ 213$ & $-\$ 181$ \\
\hline$P$ value & 0.006 & 0.253 & $<0.001$ & 0.229 & 0.112 \\
\hline
\end{tabular}

avalues are health plan-paid costs

${ }^{b}$ Mean costs for TIO + OLO cohort minus mean costs for TT cohort.

$\mathrm{COPD}=$ chronic obstructive pulmonary disease; $\mathrm{ED}=$ emergency department; $T I O+O L O=$ tiotropium/olodaterol; $T T=$ triple therapy.

The Kaplan-Meier analysis of time to first severe COPD exacerbation, any COPD exacerbation, pneumonia, and pneumonia or acute bronchitis/bronchiolitis diagnosis also favored the LAMA + LABA cohort (all, $P<0.02$ ), with a lesser proportion experiencing these events (severe COPD exacerbation: $11.5 \%$ vs. $16.3 \%$; any exacerbation: $43.4 \%$ vs. $51.4 \%$; pneumonia: $17.3 \%$ vs. $23.1 \%$; pneumonia or acute bronchitis/bronchiolitis: $25.4 \%$ vs. $31.8 \%)$.

\section{Discussion}

Among rigorously matched cohorts who were followed for the duration of patients who remained on their index treatment, mean annualized cost savings to health plans for TIO + OLO averaged $\$ 7,041$ for total COPD-related costs and $\$ 9,221$ for all-cause costs compared with TT. When the TIO+OLO cohort was expanded to include any LAMA + LABA regimen, similar, albeit slightly lower, annualized total cost savings were observed for LAMA+LABA over TT, with differences of
$\$ 6,412$ for COPD-related costs and $\$ 8,071$ for all-cause costs The current study builds on our recent real-world study that compared TIO + OLO with TT among newly treated MAPD plan beneficiaries for 12 months using an intent-to-treat approach and found mean annual plan savings of $\$ 4,118$ and $\$ 5,384$ for COPD-related costs and all-cause total costs, respectively, among patients on $\mathrm{TIO}+\mathrm{OLO} .{ }^{21}$ The higher savings for $\mathrm{TIO}+\mathrm{OLO}$ (and LAMA + LABA) observed in the current study likely are due to the on-treatment methodology that provides a more sensitive comparison of therapies.

Savings in COPD-related medical costs for TIO+OLO contributed $52.1 \%(\$ 3,666)$ of the difference in COPD-related total costs between cohorts. This was primarily driven by reduced acute inpatient costs (83.3\%) and aligns with the cohort differences for severe COPD exacerbations. The time to first severe COPD exacerbation was longer for TIO + OLO, with fewer patients diagnosed $(9.0 \%$ for TIO + OLO vs. $16.1 \%$ 


\section{FIGURE 2 Post-Match Annualized COPD/Pneumonia-Related Medical Costs During Follow-up} with $\mathrm{TIO}+\mathrm{OLO}$ vs. TT

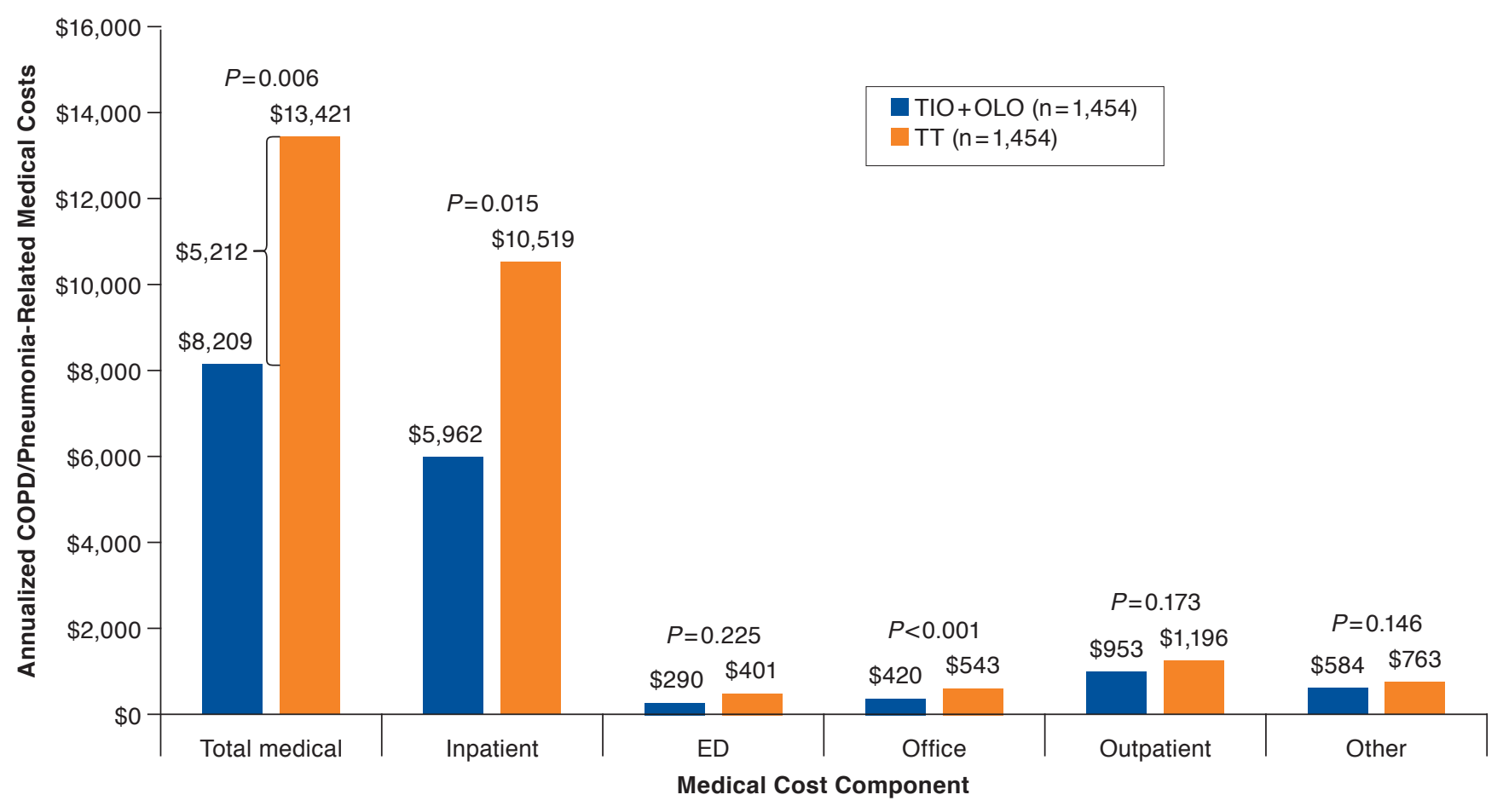

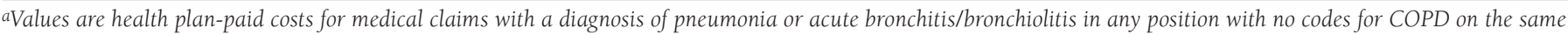
claim.

COPD = chronic obstructive pulmonary disease $; E D=$ emergency department; TIO +OLO = tiotropium/olodaterol; TT = triple therapy .

for TT); these results also confirm our previous findings. ${ }^{21}$ The pattern of lower acute inpatient costs and longer time to first COPD exacerbation (any, severe) was also observed for LABA + LAMA versus TT. While there was a trend for reduced COPD exacerbations of any severity for $\mathrm{TIO}+\mathrm{OLO}$ versus TT, the results did not achieve statistical significance, likely due to the TIO + OLO cohort's smaller sample size.

These exacerbation findings differ from a recent metaanalysis of clinical trials that compared TT with LAMA + LABA (both in a single combination inhaler) and found a reduced rate of exacerbations (moderate or severe) with $\mathrm{TT}^{30}$ This may be attributable to differences in the patient populations-a direct function of the trials' inclusion criteria. ${ }^{31}$ All clinical trial participants had symptomatic COPD, with the majority having a history of exacerbations. ${ }^{30}$ Although we could not assess symptom burden in our study, only $41.7 \%$ and $46.2 \%$ of the $\mathrm{TIO}+\mathrm{OLO}$ and TT patients, respectively, had baseline exacerbations, indicating that disease severity differences may account for the disparity in exacerbation results observed between real-world versus clinical trial settings.
COPD-related pharmacy costs accounted for $47.9 \%(\$ 3,374)$ of the savings in total COPD-related costs for TIO+OLO vs TT. This may be driven by the use of a single inhaler to deliver $\mathrm{TIO}+\mathrm{OLO}$ while $>99 \%$ of TT patients used multiple inhalers. COPD-related pharmacy cost savings were similar for LAMA+LABA $(\$ 3,308 ; 51.6 \%)$, with $97 \%$ of LAMA + LABA patients on fixed-dose single inhalers versus $<1 \%$ of the TT cohort. Nevertheless, COPD-related pharmacy costs encompass other COPD medications (e.g., rescue inhalers), and the potential impact of the new single inhaler TT entrant on pharmacy costs will depend on its usage, pricing, and other factors.

Lower respiratory tract infections such as pneumonia not only add to the economic burden of COPD but also precipitate the majority of COPD exacerbations. ${ }^{32}$ Furthermore, patients with COPD who develop pneumonia are more likely to die as a result of pneumonia than their non-COPD counterparts. ${ }^{33}$ Thus, the risk of pneumonia should be a key consideration in selecting appropriate COPD maintenance therapy. When accounting for pneumonia/bronchitis, the annualized COPDrelated medical costs increased by $27.3 \%$ in the $\mathrm{TIO}+\mathrm{OLO}$ 


\section{Differences in Real-World Health and Economic Outcomes Among Patients with COPD Treated with Combination Tiotropium/Olodaterol Versus Triple Therapy}

\section{FIGURE 3 Post-Match Kaplan-Meier Probability of Time to First COPD Exacerbation (Any or Severe) During}

Follow-up with TIO+OLO vs. TT

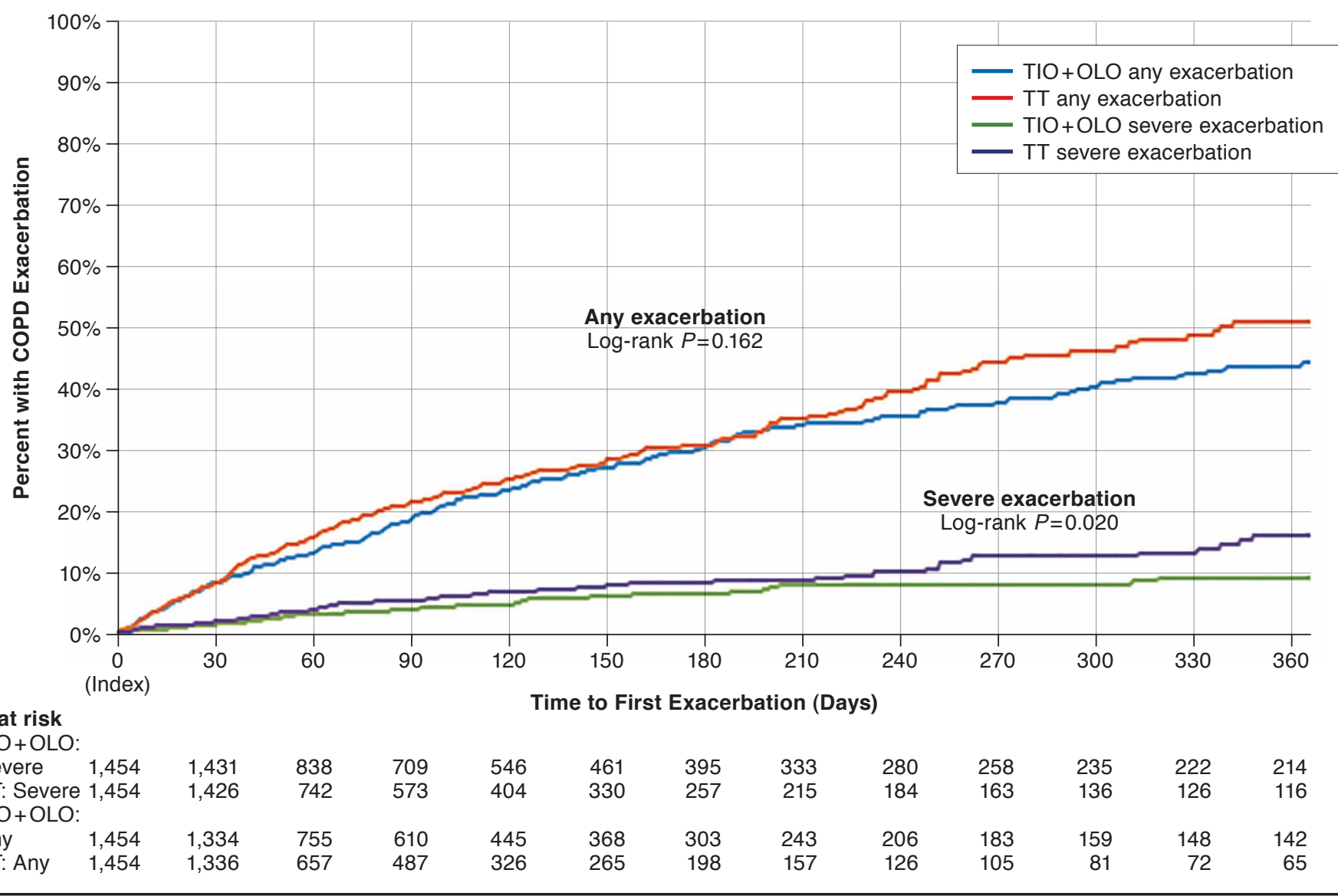

$\mathrm{COPD}=$ chronic obstructive pulmonary disease; $\mathrm{TIO}+\mathrm{OLO}=$ tiotropium/olodaterol; $\mathrm{TT}=$ triple therapy.

cohort and $32.7 \%$ in the TT cohort, resulting in additional cost savings of $22.0 \%(\$ 1,546)$ for $\mathrm{TIO}+\mathrm{OLO}$. The lesser pneumonia burden for $\mathrm{TIO}+\mathrm{OLO}$ is supported by the longer time to first pneumonia/bronchitis diagnosis and a smaller percentage of patients diagnosed (22.8\% vs. $28.4 \%$ ). Results were very similar for LAMA + LABA compared with TT and are consistent with the increased risk of pneumonia observed in clinical trials. ${ }^{30}$

Our results are consistent with prior research documenting overprescribing of TT in real-world clinical practice. ${ }^{12-15}$ The 2018 GOLD recommendations reserve TT for the most severe patients: those in Group D who experience further exacerbations and/or inadequate symptom control after dual bronchodilator therapy or, in some cases, LABA + ICS. ${ }^{5}$ Nevertheless, only $46.2 \%$ (59.4\% prematch) of patients in our TT cohort had a baseline history of exacerbations (of any type), and only $21.5 \%$ (38.7\% before matching) could be considered at high risk for exacerbations, placing them in GOLD Groups C or D. While we could not differentiate Groups $C$ and D, even assuming that all patients with a high exacerbation risk were also highly symptomatic (i.e., Group D), then roughly 60\%-80\% of patients received GOLD-discordant TT. This is consistent with previously reported ranges of TT overprescribing (64.0\%-65.9\%) observed in a similar COPD MAPD plan population. ${ }^{21}$ After applying the per-patient per-year cost differential in COPD/pneumonia-related burden between the cohorts $(\$ 9,147$ for TIO+OLO and $\$ 20,080$ for TT) for the subset of TT patients with low exacerbation risk (i.e., Groups A and $\mathrm{B}$ in this analysis population $[\mathrm{N}=1,141])$, we estimate that switching these TT patients to TIO + OLO could save health plans annually $\sim \$ 12.5$ million (\$8.7 million in medical) in total COPD-related expenditures.

GOLD recommendations limiting TT to patients with high exacerbation risk have been relatively constant over the 


\section{Differences in Real-World Health and Economic Outcomes Among Patients with COPD Treated with Combination Tiotropium/Olodaterol Versus Triple Therapy}

\section{FIGURE 4 Post-Match Kaplan-Meier Probability of Time to First Pneumonia Diagnosis ${ }^{a}$ During Follow-up}

with $\mathrm{TIO}+\mathrm{OLO}$ vs. TT

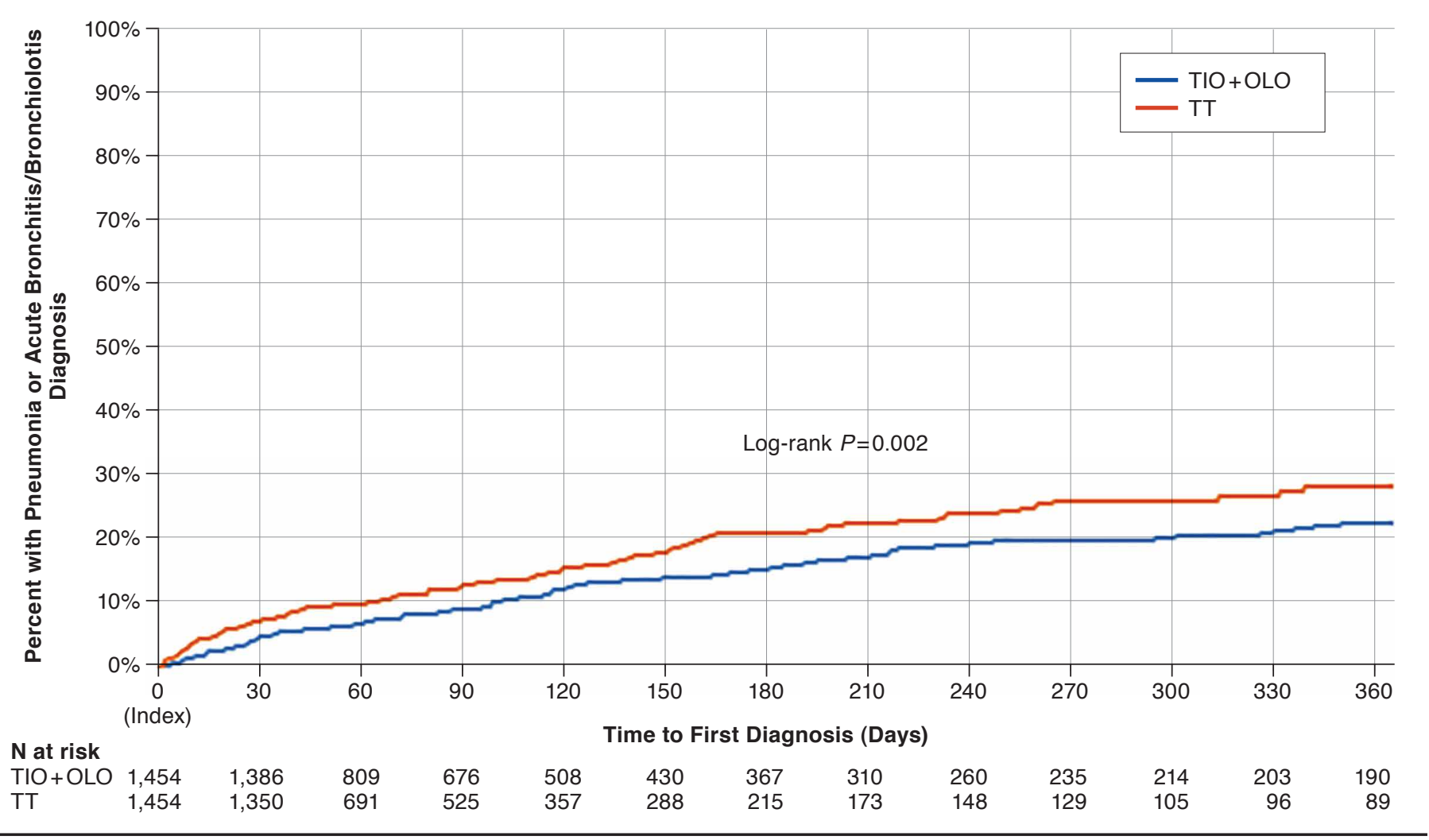

${ }^{a}$ Medical claims with pneumonia or acute bronchitis/bronchiolitis diagnosis.

$\mathrm{TIO}+\mathrm{OLO}=$ tiotropium/olodaterol; $T \mathrm{~T}=$ triple therapy.

years, and both GOLD 2018 and 2020 recommend TT only as an escalation step from LAMA+LABA or LABA+ICS, ${ }^{5,8}$ Nevertheless, $90.1 \%$ (60.0\% prematch) of TT-treated patients in this study had no baseline exposure to LAMAs and/or LABAs. The reasons for overprescribing of TT in this study and others are unknown, but possible explanations for noncompliance with GOLD recommendations include lack of clinician awareness or understanding of GOLD recommendations. ${ }^{12-15,34,35}$ Further, asthma and COPD share some common features, and ICS is the mainstay of asthma pharmacotherapy ${ }^{36}$; although we excluded patients with asthma from our study, misclassification of COPD as asthma in general practice or a consideration of asthma/COPD coexistence could result in overuse of ICS. ${ }^{37}$

Previous research in COPD populations has shown that treatment with ICS-containing regimens, most commonly $\mathrm{LABA}+\mathrm{ICS}$, is the most common precursor to TT and that overuse of ICS-containing regimens is a major cause of the deviation from GOLD recommendations. ${ }^{38,39}$ The GOLD 2019/2020 reports provide the strongest limitations on ICS-containing therapies to date: LABA+ICS as an initial therapy is considered only for patients in Group D with a history of/with concomitant asthma or elevated blood eosinophils, and TT is not recommended for initial management. ${ }^{8,40}$ Further, their follow-up recommendations limit TT to only those with persistent dyspnea post-LABA+ICS therapy or patients with further exacerbations and elevated blood eosinophil counts post-LAMA+LABA therapy. ${ }^{8,40}$ These results underscore the need for additional research to understand the rationale behind overprescribing of TT and a renewed and urgent emphasis on clinician education to align real-world prescribing patterns with GOLD recommendations, especially given the recent availability of single-inhaler TT.

\section{Limitations}

Claims-based studies have inherent limitations, including miscoding and omissions. They also lack some clinically relevant characteristics, such as symptom burden, lung function, smoking status, and blood eosinophil count. We did not 
distinguish between initial treatment at COPD diagnosis and escalation pathway. Similar to TT overprescribing, a majority of $\mathrm{TIO}+\mathrm{OLO}$ and LAMA+LABA patients $(90 \%$ prematch and $>83 \%$ postmatch) had no baseline exposure to LAMA or LABA. This suggests potential overprescribing of dual therapy as per GOLD recommendations. ${ }^{5,8}$

Although we matched patients 1:1 using a restrictive standard for matching within narrow caliper bands, modest but significant cohort differences for 4 exacerbation characteristics remained between $\mathrm{TIO}+\mathrm{OLO}$ and TT post-PSM. However, the standardized difference for these characteristics was $<11 \%$, indicating a rigorous match, given that $20 \%$ is the accepted threshold. ${ }^{41}$ We also note that the most pronounced differences in Kaplan-Meier severe COPD exacerbation curves between $\mathrm{TIO}+\mathrm{OLO}$ (and LAMA+LABA) versus TT began after $\sim 7$ months on therapy, at which point $23 \%$ and $15 \%$ of the at-risk $\mathrm{TIO}+\mathrm{OLO}$ and TT cohorts were retained. This may reflect the benefit of dual therapy's longer treatment duration or worsening outcomes with TT, but additional research is warranted into this subset.

Further, we did not assess index regimen adherence. Most TT patients used two inhalers while the TIO+OLO cohort (and $97 \%$ of LAMA+LABA) used a single inhaler. Although a clinical trial found no difference in exacerbations for TT delivered by 1 versus 2 inhalers, ${ }^{42}$ real-world results may differ. Next, our definition of therapy switch and discontinuation relied on outpatient pharmacy claims. Any patients who may have switched to a nebulized delivery method would have prompted premature censoring of the patient's follow-up. ${ }^{43}$ Finally, our results may not be generalizable to nonmanaged Medicare beneficiaries, patients with other forms of insurance, or the uninsured.

\section{Conclusions}

Annualized costs savings to health plans for patients initiating $\mathrm{TIO}+\mathrm{OLO}$ averaged $\$ 7,041$ and $\$ 8,586$ for COPD-related and COPD/pneumonia-related costs compared with TT. The first occurrence of severe COPD exacerbation and pneumonia diagnosis during follow-up took a significantly longer time for the $\mathrm{TIO}+\mathrm{OLO}$ cohort. Broadening the $\mathrm{TIO}+\mathrm{OLO}$ cohort to include any LAMA+LABA regimen resulted in slightly lower but similar costs savings and exacerbation and pneumonia outcome benefits. These real-world findings highlight the lower economic and clinical burden of TIO + OLO (or LAMA + LABA) relative to TT and provide new context for prescribing according to GOLD recommendations while also presenting evidence indicating the persistent inconsistency between the recommendations and real-world clinical practices pertaining to TT.

\section{Authors}

SWETHA R. PALLI, MS, and JESSICA FRANCHINO-ELDER, $\mathrm{PhD}$, Boehringer Ingelheim Pharmaceuticals, Inc., Ridgefield, Connecticut. MONICA FRAZER, PhD; MARY DUCHARME, MLIS; AMI R. BUIKEMA, MPH; and AMY J. ANDERSON, MS, Optum, Eden Prairie, Minnesota.

AUTHOR CORRESPONDENCE: Swetha R. Palli, MS, Boehringer Ingelheim Pharmaceuticals, Inc., 900 Ridgebury Rd., AOB 1C, Ridgefield, CT 06877. Tel.: 203.798.4460;

Email: swetha.palli@boehringer-ingelheim.com.

\section{DISCLOSURES}

This study was sponsored by Boehringer Ingelheim Pharmaceuticals, Inc. (BIPI). Palli and Franchino-Elder are employees of BIPI. Frazer, DuCharme, Buikema, and Anderson are employees of Optum, which was contracted by BIPI to conduct this study. The authors received no direct compensation related to the development of the manuscript. BIPI was given the opportunity to review the manuscript for medical and scientific accuracy as well as intellectual property considerations.

\section{ACKNOWLEDGMENTS}

The authors thank the following study team members of Optum: Stephanie Korrer, Kimberly Mcniff, and Lee Brekke for analytic support; James Hartje, Damon Van Voorhis, Vincent Peichel, and Feng Cao for programming support; and Megan Sipper and Sharanya Murali for project management. Sarah Peirce-Sandner provided medical writing assistance and was funded by BIPI.

\section{REFERENCES}

1. Sullivan J, Pravosud V, Mannino DM, Siegel K, Choate R, Sullivan T. National and state estimates of COPD morbidity and mortality - United States, 2014-2015. Chronic Obstr Pulm Dis. 2018;5(4):324-33.

2. Ford ES, Murphy LB, Khavjou O, Giles WH, Holt JB, Croft JB. Total and state-specific medical and absenteeism costs of COPD among adults aged

$\geq 18$ years in the United States for 2010 and projections through 2020. Chest. 2015;147(1):31-45.

3. Kumbhare SD, Beiko T, Wilcox SR, Strange C. Characteristics of COPD patients using United States emergency care or hospitalization. Chronic Obstr Pulm Dis. 2016;3(2):539-48.

4. Singh JA, Yu S. Utilization due to chronic obstructive pulmonary disease and its predictors: a study using the U.S. National Emergency Department Sample (NEDS). Respir Res. 2016;17:1.

5. Global Initiative for Chronic Obstructive Lung Disease, Inc. Global strategy for the diagnosis, management, and prevention of chronic obstructive pulmonary disease (2018 report). Available at: https://goldcopd. org/wp-content/uploads/2017/11/GOLD-2018-v6.0-FINAL-revised-20-Nov_ WMS.pdf. Accessed March 23, 2020.

6. Yawn BP, Li Y, Tian H, Zhang J, Arcona S, Kahler KH. Inhaled corticosteroid use in patients with chronic obstructive pulmonary disease and the risk of pneumonia: a retrospective claims data analysis. Int J Chron Obstruct Pulmon Dis. 2013;8:295-304.

7. Zheng Y, Zhu J, Liu Y, et al. Triple therapy in the management of chronic obstructive pulmonary disease: systematic review and meta-analysis. BMJ. 2018;363:k4388. 


\section{Differences in Real-World Health and Economic Outcomes Among Patients with COPD Treated with Combination Tiotropium/Olodaterol Versus Triple Therapy}

8. Global Initiative for Chronic Obstructive Lung Disease, Inc. Global strategy for the diagnosis, management, and prevention of chronic obstructive pulmonary disease (2020 report). Available at: https://goldcopd. org/wp-content/uploads/2019/11/GOLD-2020-REPORT-verl.0wms.pdf. Accessed May 15, 2020.

9. George L, Brightling CE. Eosinophilic airway inflammation: role in asthma and chronic obstructive pulmonary disease. Ther Adv Chronic Dis. 2016;7(1):34-51.

10. Singh D, Kolsum U, Brightling CE, et al. Eosinophilic inflammation in COPD: prevalence and clinical characteristics. Eur Respir J. 2014;44(6): 1697-700.

11. Pavord ID, Lettis S, Locantore N, et al. Blood eosinophils and inhaled corticosteroid/long-acting beta-2 agonist efficacy in COPD. Thorax. 2016;71(2):118-25

12. Casas A, Montes de Oca M, Menezes AM, et al. Respiratory medication used in COPD patients from seven Latin American countries: the LASSYC study. Int J Chron Obstruct Pulmon Dis. 2018;13:1545-56.

13. Ding B, Small M, Holmgren U. A cross-sectional survey of current treatment and symptom burden of patients with COPD consulting for routine care according to GOLD 2014 classifications. Int J Chron Obstruct Pulmon Dis. 2017;12:1527-37.

14. Hurst JR, Dilleen M, Morris K, Hills S, Emir B, Jones R. Factors influencing treatment escalation from long-acting muscarinic antagonist monotherapy to triple therapy in patients with COPD: a retrospective THINdatabase analysis. Int J Chron Obstruct Pulmon Dis. 2018;13:781-92.

15. Safka KA, Wald J, Wang H, McIvor L, McIvor A. GOLD stage and treatment in COPD: a 500 patient point prevalence study. Chronic Obstr Pulm Dis. 2016;4(1):45-55.

16. Lin J, Li Y, Tian H, et al. Costs and health care resource utilization among chronic obstructive pulmonary disease patients with newly acquired pneumonia. Clinicoecon Outcomes Res. 2014;6:349-56.

17. Buhl R, Criee CP, Kardos P, et al. Dual bronchodilation vs triple therapy in the "real-life" COPD DACCORD study. Int J Chron Obstruct Pulmon Dis. 2018;13:2557-68

18. Stiolto Respimat (tiotropium bromide and olodaterol) inhalation spray for oral inhalation use. Boehringer Ingelheim Pharmaceuticals. 2019. Available at: https://docs.boehringer-ingelheim.com/Prescribing\%20 Information/PIs/Stiolto\%20Respimat/stiolto.pdf. Accessed June 24, 2020

19. Palli SR, Buikema AR, Korrer S, et al. Comparing healthcare costs in COPD patients initiating tiotropium/olodaterol vs. triple therapy. Poster presented at: Academy of Managed Care Pharmacy (AMCP) Nexus; October 22-25, 2018; Orlando, Florida.

20. Palli SR, Kaila S, Juday T. Comparing healthcare cost and resource utilization in COPD patients initiating tiotropium/olodaterol (T/O) vs. triple therapy (TT). Poster presented at: 40th Annual Meeting of the Society for Medical Decision Making (SMDM); October 14-17, 2018; Montreal, Canada.

21. Palli SR, Buikema AR, DuCharme M, Frazer M, Kaila S, Juday T. Costs, exacerbations and pneumonia after initiating combination tiotropium olodaterol versus triple therapy for chronic obstructive pulmonary disease. J Comp Eff Res. 2019;8(15):1299-316.

22. Mueller S, Wilke T, Bechtel B, Punekar YS, Mitzner K, Virchow JC. Nonpersistence and non-adherence to long-acting COPD medication therapy: a retrospective cohort study based on a large German claims dataset. Respir Med. 2017;122:1-11.

23. Wurst KE, Punekar YS, Shukla A. Treatment evolution after COPD diagnosis in the UK primary care setting. PLoS One. 2014;9(9):e105296.

24. Lipson DA, Criner G, Day NC, et al. Reduction in the risk of all-cause mortality with fluticasone furoate/umeclidinium/vilanterol compared to umeclidinium/vilanterol in IMPACT including previously missing or censored vital status data. Poster presented at: American Thoracic Society International Conference; May 17-22, 2019; Dallas, Texas.

25. Jinjuvadia C, Jinjuvadia R, Mandapakala C, Durairajan N, Liangpunsakul S, Soubani AO. Trends in outcomes, financial burden, and mortality for acute exacerbation of chronic obstructive pulmonary disease (COPD) in the United States from 2002 to 2010. COPD. 2017;14(1):72-79.
26. Strange C, Walker V, DePietro M, et al. Patient-reported outcomes of dual bronchodilator fixed-dose combination versus bronchodilator monotherapy in individuals with COPD. Int J Chron Obstruct Pulmon Dis. 2019;14:1377-88.

27. Quan H, Li B, Couris CM, et al. Updating and validating the Charlson comorbidity index and score for risk adjustment in hospital discharge abstracts using data from 6 countries. Am J Epidemiol. 2011;173(6):676-82.

28. van Walraven C, Austin PC, Jennings A, Quan H, Forster AJ. A modification of the Elixhauser comorbidity measures into a point system for hospital death using administrative data. Med Care. 2009;47(6):626-33.

29. U.S. Bureau of Labor Statistics. Consumer Price Index. Medical care. Series ID: CUUR0000SAM. Available at: http://data.bls.gov/cgi-bin/ surveymost?cu. Accessed June 24, 2020

30. Zayed Y, Barbarawi M, Kheiri B, et al. Triple versus dual inhaler therapy in moderate-to-severe COPD: a systematic review and meta-analysis of randomized controlled trials. Clin Respir J. 2019;13(7):413-28.

31. Buhl R, Criee CP, Kardos P, et al. Patients in clinical trials on COPD triple therapy compared to real world populations. Poster presented at: American Thoracic Society 2019 International Conference; May 17-22, 2019; Dallas, Texas.

32. Sapey E, Stockley RA. COPD exacerbations . 2: aetiology. Thorax. 2006;61(3):250-58.

33. Restrepo MI, Mortensen EM, Pugh JA, Anzueto A. COPD is associated with increased mortality in patients with community-acquired pneumonia Eur Respir J. 2006;28(2):346-51.

34. Grewe FA, Sievi NA, Bradicich M, et al. Compliance of pharmacotherapy with GOLD guidelines: a longitudinal study in patients with COPD. Int J Chron Obstruct Pulmon Dis. 2020;15:627-35.

35. Perez X, Wisnivesky JP, Lurslurchachai L, Kleinman LC, Kronish IM. Barriers to adherence to COPD guidelines among primary care providers. Respir Med. 2012;106(3):374-81.

36. Global Initiative for Asthma. Global strategy for asthma management and prevention (2020 report). Available at: https://ginasthma.org/ wp-content/uploads/2020/04/GINA-2020-full-report_-final-_wms.pdf. Accessed May 18, 2020.

37. Miravitlles M, Garcia-Sidro P, Fernandez-Nistal A, Buendia MJ, Espinosa de los Monteros MJ, Molina J. Course of COPD assessment test (CAT) and clinical COPD questionnaire (CCQ) scores during recovery from exacerbations of chronic obstructive pulmonary disease. Health Qual Life Outcomes. 2013;11:147.

38. Brusselle G, Price D, Gruffydd-Jones K, et al. The inevitable drift to triple therapy in COPD: an analysis of prescribing pathways in the UK. Int J Chron Obstruct Pulmon Dis. 2015;10:2207-17.

39. Ghosh S, Anderson WH, Putcha N, et al. Alignment of inhaled chronic obstructive pulmonary disease therapies with published strategies. Analysis of the Global Initiative for Chronic Obstructive Lung Disease recommendations in SPIROMICS. Ann Am Thorac Soc. 2019;16(2):200-08.

40. Global Initiative for Chronic Obstructive Lung Disease. Global strategy for the diagnosis, management, and prevention of chronic obstructive pulmonary disease (2019 report). Available at: https://goldcopd.org/ wp-content/uploads/2018/11/GOLD-2019-vl.7-FINAL-14Nov2018-WMS. pdf. Accessed March 23, 2020.

41. Austin PC. An introduction to propensity score methods for reducing the effects of confounding in observational studies. Multivariate Behav Res. 2011;46(3):399-424

42. Vestbo J, Papi A, Corradi M, et al. Single inhaler extrafine triple therapy versus long-acting muscarinic antagonist therapy for chronic obstructive pulmonary disease (TRINITY): a double-blind, parallel group, randomised controlled trial. Lancet. 2017:389(10082):1919-29.

43. Tashkin DP. A review of nebulized drug delivery in COPD. Int J Chron Obstruct Pulmon Dis. 2016;11:2585-96. 